\title{
Improving foam stability and lautering conditions
}

\author{
Petr Košin ${ }^{1 *}$, Jan Šavel ${ }^{1}$, Adam Brož ${ }^{1}$, Jakub Vlček ${ }^{1}$ \\ Budějovický Budvar, n.p., Karolíny Světlé 4, \\ 37021 České Budějovice, Czech Republic
}

* corresponding author: petr.kosin@budvar.cz

\begin{abstract}
How to increase foam stability without adding foam stabilizers? As we published earlier, there was more than a sufficient amount of foam stabilizing substances in beer, but these must not be pushed out of the beer surface by foam negative substances. There are several separation steps in a brewing process: lautering, trub separation, and beer filtration; all of these can also separate foam negative substances. This short article discusses how a pH during sparging can influence foam stability. It seems that the lower the $\mathrm{pH}$, the fewer foam negative substances are carried over to the following production steps of a brewing process.
\end{abstract}

Keywords: foam stability, lautering, foam negative components

\section{Introduction}

There are foam positive and foam negative substances in beer. The former are well known, but difficult to control when a recipe of a beer brand is to remain unchanged. We have published results proving that there is a sufficient amount of foam positive substances in any beer and that foam stability would not be a problem if these substances did not have to fight for their position on the beer surface with foam negative substances (Kosin et al., 2010). The only question is how to lower the amount of foam negative substances without changing a brand recipe.

There are several separation steps in a brewing process. We discussed the possibility of separating foam negative substances by using absorbents during beer filtration (Kosin et al., 2018). This technique is still under development. The second major separation step in a brewing process is lautering. Its capability to separate foam negative components will be shortly discussed in this paper. We mainly focused on the $\mathrm{pH}$ during sparging, as the $\mathrm{pH}$ strongly influences solubility of many foam negative compounds.

\section{Material and methods}

Pale lager beer (5.0\% alcohol by volume) was brewed from soft water, pale malt and Saaz hop cones by the classical Czech double decoction mashing, lautering in a lauter tun was followed by sparging until the extract decreased under $1 \%$ and consequently by a two phase fermentation and maturation technique.

Lacticacid was of food grade quality $\left(80 \%, 1.209 \mathrm{~kg} . \mathrm{L}^{-1}\right)$ and was added to sparging water. Control batches were sparged with standard soft brewing water.

A Matrix Foaming Potential (MFP) was measured with a Foam Stability Tester (Figure 1, 1-CUBE, Havlickuv Brod, Czech Republic). The sample was first degassed (if carbonated) by gentle shaking until no further foam was formed by bubble nucleation. The foam was prepared with a foam stability mixer with a medium flow rate of air (0.5 L.min ${ }^{-1}$ ) and a mixer revolution speed of $900 \mathrm{rpm}$. Foam stability was evaluated by electrode sensors as the time taken for the foam surface to decrease to a point of $5 \mathrm{~mm}$ above the beer surface.
Research Institute of Brewing and Malting, Plc. Published online: 15 April 2020 
NIBEM foam stability values were measured with a NIBEM TPH foam stability tester (Haffmans BV, Venlo, The Netherlands) according to the protocol recommended by the manufacturer.

A pouring test of foam stability was evaluated by standard pouring of beer into a beer glass and measurement of time to bald patch larger than $5 \mathrm{~mm}$ on the beer surface.

\section{Results and discussion}

\subsection{Matrix Foaming Potential (MFP) during lautering}

Sweet wort and run offs were sampled during the course of a production scale (600 hl batch) lautering and sparging. The MFP decreased rapidly during sparging together with an increase of the $\mathrm{pH}$ (Figure 1). Such a decrease is not proportional to the decrease of pure wort extract concentration. The decrease of the MFP during sparging is caused by an increase of a foam negative compounds content, which is proved by the MFP of beer with minor addition (3\%) of last run-offs (Figure 2).

\subsection{Alternating sparging conditions}

Addition of $0.1 \%$ of lactic acid into the sparging water increased significantly the MFP of sweet and hopped wort in laboratory sparging (Figure 3). More importantly, an addition of run-offs sparged at a lower $\mathrm{pH}$ to the beer did not decrease the MFP of beer, which means that these run-offs contained a lower amount of foam negative components (Figure 4). We assume that fatty acids had a lower amount of dissociated carboxyl groups at a lower $\mathrm{pH}$, which could cause a lower solubility of fatty acids in run-offs and preserve their presence in the spent grains.
Figure 1 Matrix foaming potential (MFP) and $\mathrm{pH}$ during lautering and sparging with water

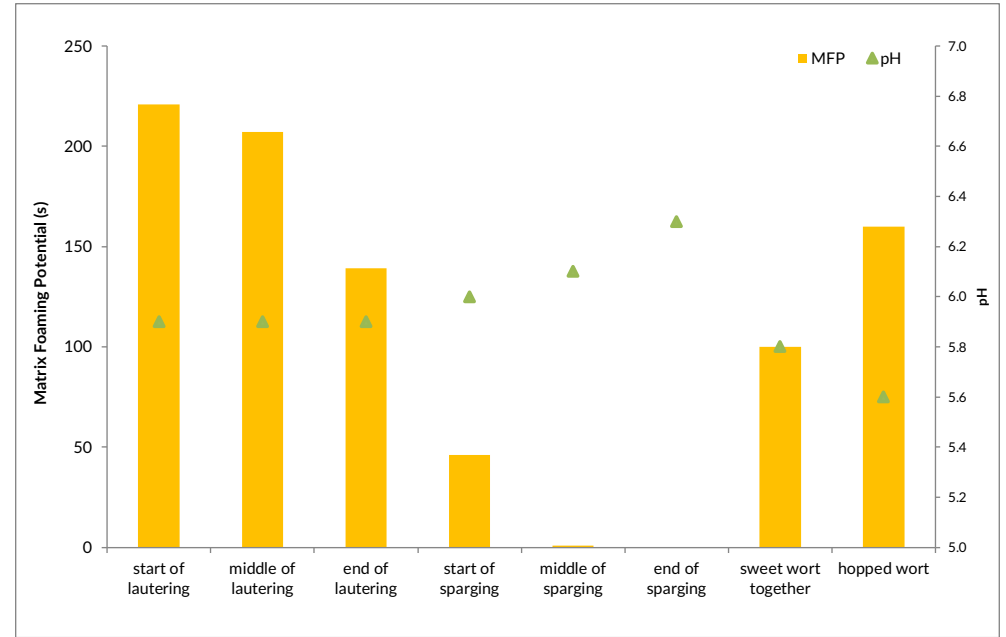

Figure 2 Matrix foaming potential of beer and beer with minor addition of last run-offs sparged with water

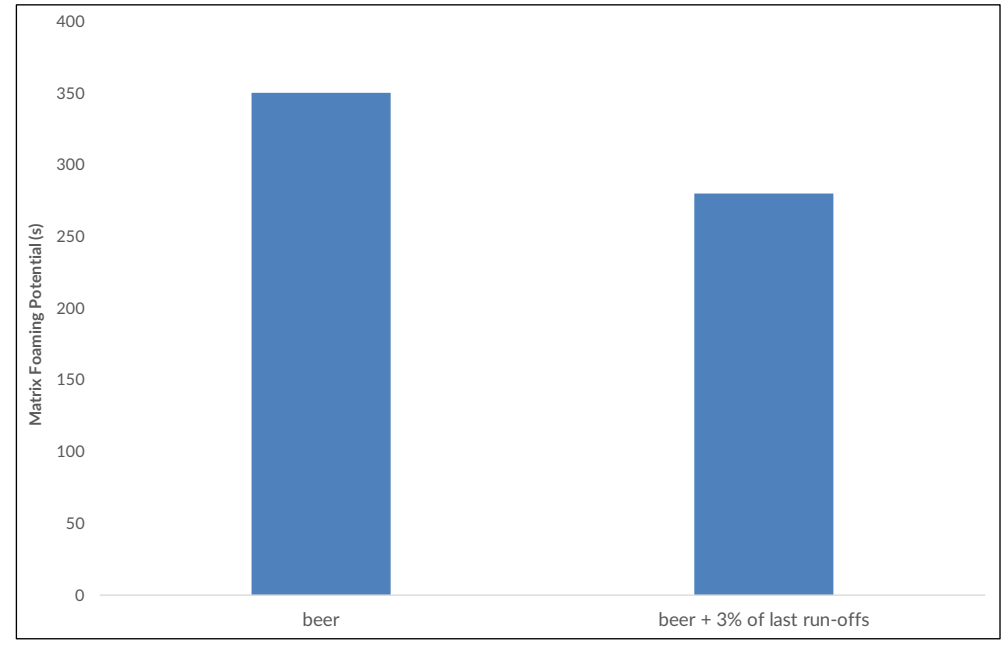

Figure 3 Matrix foaming potential (MFP) and $\mathrm{pH}$ during lautering and sparging with $0.1 \%$ lactic acid

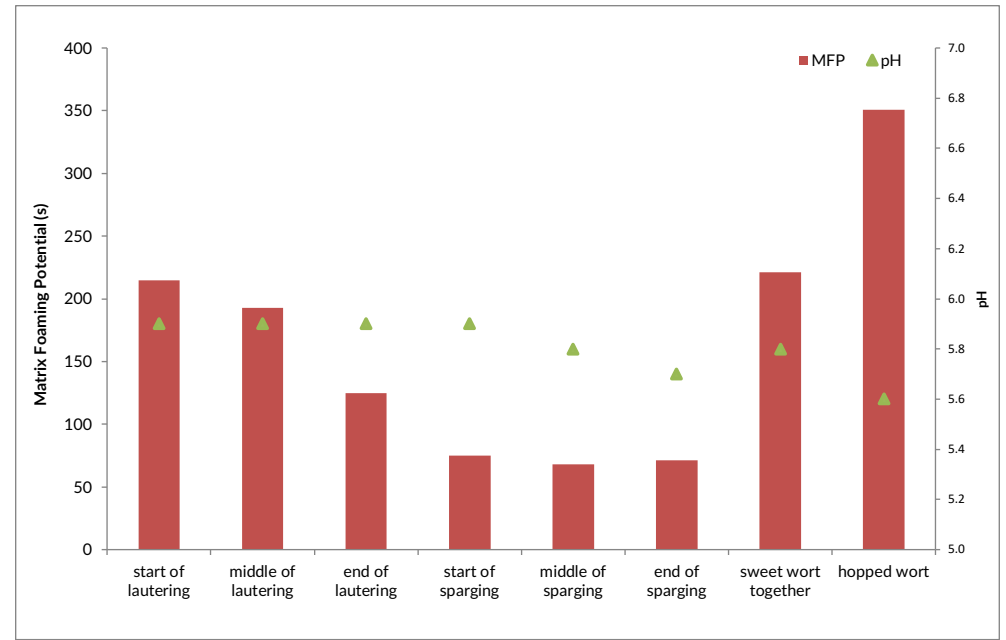




\subsection{Foam stability and other quality pa- rameters of beer sparged at lower $\mathrm{pH}$}

The beer was produced on an industrial scale according to a standard recipe, with the exception that $0.1 \%$ lactic acid solution was used as sparging liquor instead of pure water. All commonly evaluated parameters of the production and the product remained unchanged (extract yield, hop acids yield, fermentability, final beer colour, $\mathrm{pH}$, etc.). The foam stability of the beer evaluated by 3 methods increased significantly (Table 1). The most significant difference was observed by the pouring test and the MFP, which reflect consumer behaviour of the beer foam. The NIBEM change was of a lower significance, but according to our experience (unpublished data) the NIBEM method is more sensitive to the change of foam positive compounds content than to foam negative compounds content. The NIBEM does not usually "see" bald patches on the beer surface, but is more sensitive to "Styrofoam-like" objects floating on the beer surface.

\section{Conclusion}

There is a possibility to set conditions during sparging so that a lower amount of foam negative components could be separated. We assume that a lower $\mathrm{pH}$ during sparging caused a lower degree of dissociation of fatty acids and therefore also a lower solubility of these in sweet wort run-offs. Increased foam stability of the beer sparged at a lower $\mathrm{pH}$ was observed.
Figure 4 Matrix foaming potential of beer and beer with minor addition of last run-offs sparged with $0.1 \%$ lactic acid

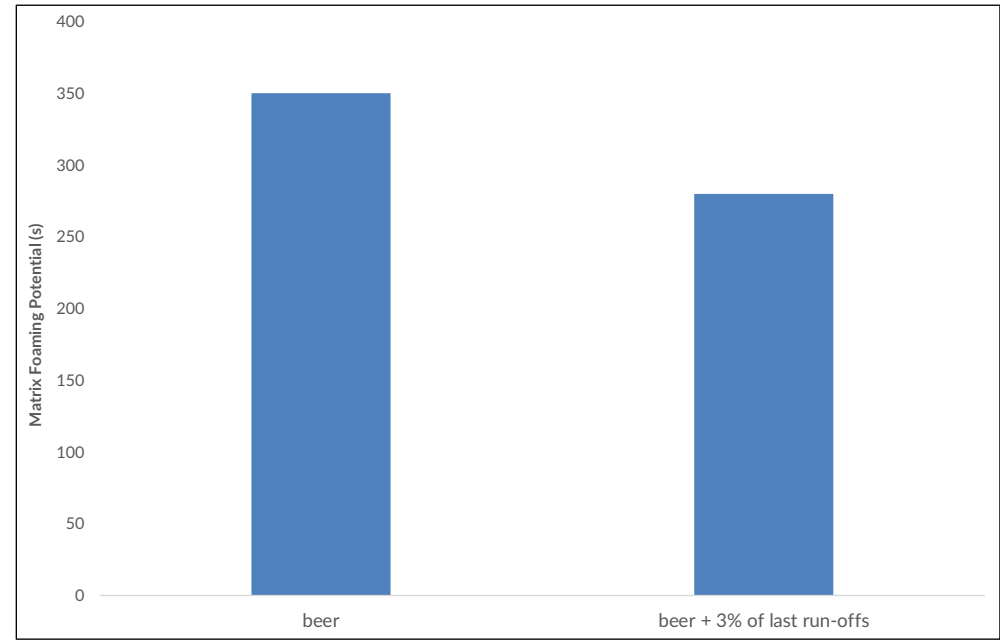

Table 1 Foam stability of beer sparged with water or $0.1 \%$ lactic acid (LA) evaluated by different methods.

\begin{tabular}{|l|c|c|}
\hline \multicolumn{1}{|c|}{ Foam stability test } & $\begin{array}{c}\text { Sparged with } \\
\text { water }\end{array}$ & $\begin{array}{c}\text { Sparged with } \\
0.1 \% \text { LA }\end{array}$ \\
\hline NIBEM (s) & 250 & 270 \\
\hline MFP (s) & 40 & 240 \\
\hline Pouring test (s) & 270 & 630 \\
\hline
\end{tabular}

\section{References}

Kosin, P., Savel, J., Evans, D. E., Broz, A. (2010): Relationship between matrix foaming potential, beer composition, and foam stability. J. Am. Soc. Brew. Chem. 68(1), 63-69. https://doi-org.ezproxy.techlib. cz/10.1094/ASBCJ-2010-0114-01

Kosin, P., Branyik, T., Savel, J., Ulmann, F., Vlcek, J. (2018): Use of sorbents to increase beer foam stability. J. Am. Soc. Brew. Chem. 76(1), 58-61. https:// doi-org.ezproxy.techlib.cz/10.1080/03610470.2017.1398565 